\section{Cahiers de Narratologie}

Analyse et théorie narratives

8 | 1997

Création de l'espace et narration littéraire

\title{
Espace et sens chez Cortázar « Reunión con un círculo rojo »
}

\section{Marie-Pierre Lavaud}

\section{(2) OpenEdition}

Journals

Édition électronique

URL : http://journals.openedition.org/narratologie/11543

DOI : 10.4000/narratologie.11543

ISSN : 1765-307X

Éditeur

LIRCES

\section{Édition imprimée}

Date de publication : 1 décembre 1997

Pagination : 209-221

ISBN : 291089746X

ISSN : 0993-8516

\section{Référence électronique}

Marie-Pierre Lavaud, «Espace et sens chez Cortázar « Reunión con un círculo rojo » », Cahiers de Narratologie [En ligne], 8| 1997, mis en ligne le 15 décembre 2020, consulté le 25 février 2021. URL http://journals.openedition.org/narratologie/11543; DOI : https://doi.org/10.4000/narratologie.11543

Ce document a été généré automatiquement le 25 février 2021.

Article L.111-1 du Code de la propriété intellectuelle. 


\title{
Espace et sens chez Cortázar «Reunión con un círculo rojo »
}

\author{
Marie-Pierre Lavaud
}

1 À la lecture de "Reunión con un círculo rojo ", conte fantastique de Cortázar publié pour la première fois en 1976 dans le recueil Alguien que anda por ahí ${ }^{1}$, nous avons été frappée par la différence entre le traitement du temps et des personnages d'une part et celui de l'espace d'autre part. Le lecteur, en effet, assiste à un gommage systématique de toute marque temporelle précise : aucune indication d'année, de saison, de jour ou d'heure. On sait simplement qu'il fait froid, qu'il pleut et que c'est plus ou moins l'heure du dîner. Les personnages sont tout aussi mystérieux. Par contre, les lieux sont, eux, clairement nommés, ce qui nous a amenée à considérer l'espace comme un élément fondamental pour appréhender le conte. Par ailleurs, outre l'espace en tant que lieu pratiqué, le conte convoque d'autres espaces signifiants par le biais de la transtextualité.

2 Nous nous intéresserons donc, dans un premier temps, à l'espace diégétique, pour nous pencher, ensuite, sur ces univers signifiants. Nous espérons montrer que ces différents espaces forment un faisceau convergent créateur de sens.

\section{L'espace diégétique}

3 Rappelons brièvement la trame du conte : un narrateur apparemment extradiégiétique s'adresse à un narrataire, Jacobo, et lui parle d'une nuit passée que ce dernier a vécu en Allemagne. Jacobo pousse la porte d'un restaurant de Wiesbaden appelé le Zagreb et une touriste anglaise entre peu après. Pressentant un danger, Jacobo décide d'attendre que la touriste ait fini de dîner pour s'en aller. Elle sort et il la suit, à la fois soulagé et inquiet. Celle-ci ayant disparu au coin d'une rue, Jacobo, persuadé qu'elle est retournée au restaurant, fait demi-tour et revient au Zagreb. Une surprise l'attend : tout est prêt pour une mystérieuse cérémonie. Une surprise attend également le lecteur, puisqu'il apprend dans le dernier paragraphe du texte que le narrateur inconnu n'est autre que la touriste anglaise qui révèle l'ironie de la situation. Jacobo s'est jeté dans la gueule du 
loup et a perdu la vie en voulant sauver d'un mystérieux danger celle qui, en fait, voulait le protéger. Ce sont maintenant deux morts-vivants qui vont hanter les nuits pluvieuses.

L'action nous entraîne donc en Allemagne, à Wiesbaden et plus exactement dans un restaurant : le Zagreb. Si ce dernier provoque d'abord chez Jacobo une réaction mitigée, « entre molestia y alivio ", il est cependant très vite présenté comme un refuge :

... el enclave transilvânico que lo protegía de la lluvia y de una ciudad alemana no excesivamente interesante. El silencio, las ausencias y la vaga luz eran ya casi sus amigos, en todo caso lo distanciaban del resto y lo dejaban hermosamente solo ...2

5 C'est un espace calme, silencieux et chaud (" lo que cuenta es el calor ») où le convive n'est pas agressé par une lumière excessive. Le choix de Jacobo de s'installer dans un coin renforce cette idée de refuge. Rappelons que le coin, pour Bachelard, est l'espace de l'être, c'est une chambre imaginaire que celui-ci construit autour de son corps afin de se protéger ${ }^{3}$. Les objets qui vont peupler ce décor correspondent à ce que l'on s'attend à trouver dans un restaurant: des tables aux nappes blanches agrémentées de bougies, un vieux portemanteau, un comptoir, des assiettes, des verres ... Tout porte à croire que les espaces nommés et décrits ne sont là que pour ancrer la fiction dans la réalité. Le choix de Wiesbaden comme cadre de l'action pourrait être un simple clin d'œil autobiographique, puisque Cortázar, dans une interview de Ernesto González Bermejo, précise que la première partie du conte est née d'une expérience personnelle vécue dans un restaurant allemand ${ }^{4}$. Les éléments spatiaux fonctionneraient donc comme de simples "informants", autrement dit des données immédiatement signifiantes permettant d'authentifier la réalité du référent. Toutefois, il est surprenant que ces informants ne soient que spatiaux. Par ailleurs, tout lecteur de Cortázar sait très bien qu'il s'applique consciencieusement à éliminer tout élément gratuit de ses textes au nom du fameux concept d'intensité5. Il nous faut donc postuler que les marques d'espace ont une valeur fortement indicielle qu'il nous faut analyser. En fait, l'espace va se révéler créateur de sens.

6 Au fil de la lecture, la perception par Jacobo de l'espace du restaurant évolue et l'atmosphère est de plus en plus pesante.

... la doble manía de cruzarse de brazos apenas terminaban su trabajo hubiera sido divertida pero de alguna manera no lo era, ...(p. 168)

Tal vez la idea de quedarse nuevamente solo en la sala, eso que había visto tan agradable al llegar y ahora era diferente,... (p. 170)

7 Les serveurs sont inquiétants, ils surgissent de nulle part (« llegó como desde la nada, dibujándose de golpe junto al mantel blanco »), sont d'une pâleur suspecte sur laquelle le texte insiste à plusieurs reprises. En outre, ils ont des gestes mécaniques d'automates :

...repitió mecánicamente café a la turca ... (p. 167)

Uno de los camareros presentaba la cuenta con un gesto de autómata, alargando el brazo y quedándose inmóvil ... (p. 170)

Un réexamen attentif des termes spatiaux confirme cette sensation. Il s'agit d'un restaurant, par conséquent d'un espace fermé. Or, si l'espace clos peut être vu comme un refuge, il représente aussi l'enfermement, l'isolement et les marques en sont nombreuses dans le texte. Rappelons, par exemple, que le Zagreb est défini comme « el enclave transilvánico». Le coin, dans sa symbolique, participe lui aussi, de cet isolement. Il marque un resserrement physique sur soi et par là même une négation de l'univers. Jacques Poulet, dans cette perspective, attire notre attention sur 
l'imperméable défini comme "una burbuja » que la touriste anglaise a tant de mal à quitter ${ }^{6}$. Il ne faudrait pas non plus oublier le terme « círculo » contenu dans le titre. Le cercle est une courbe fermée dont on ne sort pas, une boucle et il faudrait le mettre en rapport avec l'itinéraire du héros qui, à la fin du texte, revient à son point de départ, le restaurant. Cette connotation insistante de l'enfermement, fait apparaitre l'espace du restaurant comme un piège et il est intéressant de souligner que le terme "peligro " apparaît explicitement dans le texte : «Sin siquiera una idea articulable olió el peligro, ...» (p. 169).

9 Cependant, rien n'est clairement dit. Que se passe-t-il dans ce restaurant ? Ce sont les notations géographiques qui vont nous donner la solution. Pourquoi, en effet, situer l'action dans un restaurant slave en Allemagne? De plus, le lecteur ne peut que relever l'insistance sur ce caractère slave: outre le nom du restaurant, il est question de " enclave transilvánico », « salón vagamente balcánico », «algún postre balcánico ». En fait, les Balkans nous renvoie ici à un espace mythique et folklorique, celui des vampires. N'oublions pas que dans Dracula, le célèbre roman de Bram Stoker qui va imposer l'archétype du vampire, le personnage principal était un comte de Transylvanie. Dans cette perspective, on peut s'interroger sur le choix d'une ville allemande. Situer l'action en Europe centrale aurait été trop évident, le choix de Wiesbaden est beaucoup plus habile. C'est, au dire du narrateur, « una ciudad alemana no excesivamente interesante»; en fait, ce qui importe, c'est que la ville soit allemande. Or c'est au réalisateur allemand Fiedrich Wilhelm Murnau que l'on doit la première adaptation cinématographique du roman de Stoker: Nosferatu oder eine Symphonie des Grauens (Nosferatu le vampire). Par ailleurs, le mot "vampire ", d'origine

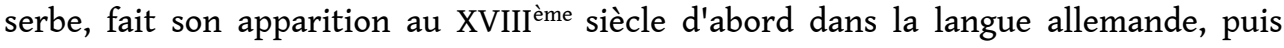
ensuite dans les autres langues occidentales. Le restaurant semble donc être un repaire de vampires. Et curieusement, fiction et autobiographie se mêlent; Cortázar en effet, raconte la chose suivante à propos de la genèse de ce conte :

Todo lo que cuento al principio del relato sucedió así. Entré a ese restaurante y tuve miedo porque llegué a la convicción absoluta de que allí había vampiros. [...] Y cuando entró aquella turista, que yo imaginé inglesa, sentí la obligación de protegerla, siendo las dos únicas personas que estábamos allí aquella noche de lluvia.

Y no me moví hasta que ella se fue, aunque - y esto pudo haber sido sugestióntuve la impresión palpable de que el personal del restaurante, la dueña y los dos camareros me odiaban por eso $^{7}$.

10 A la lueur de ces précisions, un certain nombre de notations font sens. Ainsi, le lecteur comprend mieux l'insistance du texte sur la pénombre ("vaga luz», "apenas iluminado ", « un restaurante ... tan como lejos de la luz ») : chacun sait que le vampire est une créature de la nuit. La couleur rouge extrêmement présente (" espeso cortinado rojo ", « una blusa entre solferino ${ }^{8} \mathrm{y}$ tomate ", " chaleco rojo », ...) et annoncée dès le titre (" círculo rojo») rappelle le vampirisme. Le teint blême des serveurs et leurs mouvements mécaniques prennent également tout leur sens: ce sont des mortsvivants, donc plus vraiment humains. Cela explique aussi le reflet trompeur dans la glace, car un vampire, normalement, ne se reflète pas dans un miroir.

Los camareros se habían situado detrás del mostrador, a los lados de la mujer, y esperaban también con los brazos cruzados, tan parecidos entre ellos que el reflejo de sus espaldas en el azogue envejecido tenía algo de falso, como una cuadruplicación difícil o engañosa, (p. 166) 
11 Ils transpercent leur victime par le regard et lui font perdre le sens des réalités. Le caractère malsain du regard des serveurs est fréquemment relevé, avec une réitération lancinante du verbe « mirar » :

... los tres miraban a la turista inglesa, la miraban como esperando algo, que llamara para completar un pedido o acaso cambiarlo o irse, la miraban de una manera que a usted le pareció demasiado intensa, en todo caso injustificada. De usted habían dejado de ocuparse, [...] la mujer tenía la cabeza un poco gacha y el largo pelo lacio le tapaba los ojos, pero acaso era la que miraba más fijamente a la turista y a usted eso le pareció desagradable y descortés [...]. Bruscamente el centro de atención parecía haber cambiado, porque también los dos camareros lo miraban beber el café, y antes de que lo terminara la mujer se acercó a preguntarle si quería otro, y usted aceptó casi perplejo en todo eso, que no era nada, había algo que se le escapaba y que hubiera querido entender mejor, (p. 167-168)

Il ressort donc de cette rapide analyse de l'espace diégétique que si les notations spatiales servent dans un premier temps à ancrer la fiction dans le réel, elles ont aussi une valeur indicielle indéniable. En convoquant l'espace mythique et folklorique du vampire, elles sont créatrices de sens. C'est ainsi que fonctionne le fantastique cortazarien: l'insolite émerge d'un contexte trivial et quotidien. Dans ce conte de vampire, les figures du cercle et de l'enfermement s'appliquent aussi bien au héros masculin qu'à la touriste anglaise, vampire-victime condamné à faire indéfiniment elle aussi de nouvelles victimes afin d'assurer sa subsistance.

\section{Les autres espaces signifiants}

Depuis Bakthine, il est admis que le texte est un véritable carrefour intertextuel où plusieurs voix coexistent. Ici, outre l'espace vampirique des Balkans, le texte fait surgir par transtextualité d'autres univers signifiants qui viennent appuyer, pour la plupart, la lecture mythique en l'enrichissant. Il nous faut préciser d'emblée que pour les besoins de l'analyse de ce conte, il nous faut prendre le terme "transtextualité » dans un sens plus large que ne l'entend Genette. Rappelons que pour Genette, il s'agit de «tout ce qui met en relation le texte, manifeste ou secrète, avec d'autres textes " ${ }^{9}$. Dans le cas de "Reunión con un círculo rojo", nous devons aussi nous intéresser aux relations qu'entretient le texte avec d'autres types de discours, et en l'occurrence ici un discours emprunté à une autre forme d'art : nous voulons parler de la peinture.

L'univers pictural, en effet, est convoqué dès le para-texte, avec la dédicace et le titre. Lorsque le conte paraît pour la première fois ${ }^{10}$, la dédicace, "A Borges ", est plutôt laconique, pour ne pas dire trompeuse. Par la suite, il sera accompagné d'une note qui efface toute ambiguïté : " este relato se incluyó en el catálogo de una exposición del pintor venezolano Jacobo Borges ». Remarquons que l'hommage au peintre conduit l'écrivain à donner à son personnage principal le prénom de l'artiste. Le titre du conte est également une référence au peintre puisqu'il est emprunté à l'un de ses tableaux et Cortázar reconnaît que celui-ci constitue le point de départ du conte :

El cuento parte de un cuadro de Borges en el que los elementos, vamos a llamarles, vampíricos, de anormalidad, de aberración humana son muy perceptibles, como en toda su obra. Los cuadros están llenos de monstruos, de misterio, de cosas extrañas que ocurren, de personajes que avanzan un poco saliendo de un cortinado, sin que se sepa muy bien lo que son, como en los cuadros de Francis bacon, que ha influido mucho en él. ${ }^{11}$ 
15 Jacobo Borges, connu pour ses personnages fantomatiques au regard vide, a donc impressionné Cortázar et lui a donné envie de retravailler un motif folklorique qui avait été au centre de la littérature fantastique du XIX ${ }^{\text {ème }}$ siècle. Soulignons au passage que le fameux cercle rouge du titre est peut-être un clin d'œil à l'écrivain anglais Jonathan Swift. Dans les Voyages de Gulliver, en effet, roman dans lequel il aborde le thème vampirique du mort en sursis, le vampire est repérable à la naissance grâce à une tache rouge circulaire sur le front au-dessus du sourcil gauche. C'est le signe infaillible que l'enfant ne mourra jamais. Le para texte, ici, serait donc une piste de plus en faveur de la lecture fantastique. Mais, faut-il s'arrêter là? Un article de Malva E. Filer nous incite à aller plus $\operatorname{loin}^{12}$. Elle nous rappelle que, lors de la publication, en 1978, de « Reunión con un círculo rojo » dans le recueil Territorios, Cortázar a assorti le texte des photos de cinq tableaux de Borges. Selon elle, le conte est le reflet verbal du tableau intitulé « La vitrina ", avec quelques retouches. Les autres tableaux choisis pour illustrer le conte, et notamment celui qui lui donne son titre, ont une nette dimension socio-politique. Une bonne part de l'œuvre de Jacobo Borges est une réflexion sur le pouvoir, avec notamment toute une galerie de portraits sinistres: le général, l'aristocrate, le prélat, le magistrat ... "Reunión con un círculo rojo », en particulier, met en scène des militaires. Malva E. Filer le décrit de la façon suivante :

El cuadro presenta a un grupo de militares de alto rango, y entre dos de ellos, incongruentemente, una mujer que se exhibe en actitud voluptuosa. El conjunto de estas figuras, sentadas frente a un espacio circular, anónimo, de color rojo, tiene una calidad estática de escena teatral inmovilizada en el tiempo. Las siluetas se ven desdibujadas, sin que se perciban rasgos individuales. Como en otros de sus cuadros, nos encontramos con rostros apenas esbozados, de mirada vacía. Borges, dice Ashton ${ }^{13}$, «logra presentar la fotografía de prensa habitual de una reunión oficial bajo un tono fantasmagórico y portentoso que encierra en su mensaje el pasado y el presente $»^{14}$.

On peut donc lire dans la peinture de Jacobo Borges une dénonciation des abus du pouvoir, de l'hypocrisie et de l'injustice. La référence à sa peinture donne alors une nouvelle dimension, socio-politique, au conte de Cortázar. Cette dimension n'est pas surprenante dans Alguien que anda por ahí qui renferme des contes comme «Segunda vez », « Apocalipsis de Solentiname » et "Alguien que anda por ahí » faisant clairement allusion aux réalités politiques latino-américaines. N'oublions pas qu'à cause de ces trois contes, le recueil avait été interdit par la junte militaire. Cela nous amène à revenir sur l'image du cercle dont nous avons parlé précédemment. On peut se demander si cet enfermement symbolisé par le cercle, n'est pas, sur le plan idéologique, une façon de dénoncer l'oppression, qui est une certaine forme de vampirisme. Malva Filer nous rappelle, à ce propos, que Cortázar a publié en 1975 un petit livre intitulé Fantomas contra los vampiros multinacionales dans lequel il dénonçait les responsable de la violation des droits de l'Homme.

Mais abandonnons le paratexte pour entrer dans le corps du texte. Là aussi, plusieurs espaces, littéraires cette fois, sont convoqués. Pour un lecteur de Cortázar, la référence la plus évidente est Cortázar lui même. On retrouve, en effet, dans « Reunión con un círculo rojo » de nombreux éléments déjà présents dans les premiers chapitres de son grand roman de vampire, 62 Modelo para armar, dont l'hypotexte est le chapitre 62 de Rayuela. Dans 62 Modelo para armar, un homme entre sans savoir très bien pourquoi, vraisemblablement pour échapper à la bruine du Quartier latin, dans le restaurant Le Polidor. Il s'isole à une table du fond (Jacobo s'installe, lui, dans un coin), commande 
une bouteille de Sylvaner (pour Jacobo, c'est « un vino espeso y fragante que nada tenía de occidental, p. 223.) et le narrateur fait une allusion à peine voilée au vampirisme en faisant remarquer la similitude phonétique entre Sylvaner et Transylvanie :

... y pedir sin reflexión previa una botella de Sylvaner, que contenía en sus primeras sílabas como en una charada las sílabas centrales de la palabra donde latía a su vez el centro geográfico de un oscuro terror ancestral, no pasaba en definitiva de una mediocre asociación fonética ${ }^{15}$.

Notre client, comme Jacobo, fume entre les plats. Pour ce qui est du décor, un grand miroir réfléchit l'image de la salle. Le restaurant est vide et peu éclairé :

... y entonces sin transición la puerta anodina y lúgubremente iluminada del restaurante Polidor, la entrevisión de una sala casi desierta envuelta en una luz que la ironía y el malhumor sólo podían calificar de cárdena ${ }^{16}$.

Par ailleurs, le personnage de Frau Marta, vampire qui soutire du sang à ses soubrettes dans les souterrains de la Blutgasse en leur arrachant des cris $^{17}$, peut permettre d'éclaircir l'allusion finale de la serveuse du Zagreb à des cris mystérieux.

... después no fueron más que los gritos, es absurdo que griten tanto (p. 173).

Il est évident que les deux espaces littéraires cohabitent et la reprise d'un certain nombre d'éléments de 62 Modelo para armar confirme bien la lecture d'une revisitation $\mathrm{du}$ thème fantastique du vampire.

Mais le rapprochement évident avec 62 Modelo para armar ne se contente pas de confirmer la piste de la lecture vampirique. On assiste parallèlement à la convocation d'un autre espace, mythologique cette fois, par le jeu sur le nom des restaurants. Dans 62 Modelo para armar, le restaurant s'appelle Le Polidor. Or Polydoros, fils de Priam et d'Hécube, est un héros mythologique au destin tragique. La légende raconte que Priam confia son jeune fils à son gendre Polymestor, roi de Thrace, ainsi que de nombreux trésors, afin qu'il puisse tenir son rang en cas de disparition de son père. Mais Polymestor tua Polydoros et l'enterra sur la côte de Thrace. Enée aborda quelque temps plus tard sur cette même côte et, désireux de décorer l'autel sur lequel il voulait sacrifier, coupa des branches des arbres qui avaient poussé sur la tombe de Polydoros. Des gouttes de sang -signe de vie-s'échappèrent alors des branches.

Dans « Reunión con un círculo rojo ", le nom du restaurant fait également référence à un héros mythologique : Zagreus. Considéré généralement comme le fils de Zeus et de Perséphone, c'est le premier Dionysos. Craignant la jalousie de Héra, Zeus confia Zagreus enfant à Apollon et aux Curètes, qui l'élevèrent dans les forêts du Parnasse. Héra chargea alors les Titans de le retrouver. Ceux-ci, profitant d'un moment d'inattention des Curètes, attirèrent l'enfant avec des jouets (dont un miroir), le tuèrent, le démembrèrent et le dévorèrent en partie cru et en partie cuit. Zeus parvint néanmoins à le ressusciter grâce à son cœur sauvé par Athéna. Le dieu Zagreus ou Dionysos est protéiforme. Enfant, il est présenté comme un être fragile ; adulte, c'est un dieu sanguinaire. Les deux espaces diégétiques ouvrent donc tous les deux la porte à un espace mythologique dans lequel nous retrouvons, dans les deux cas, le thème de l'être sans défense confié à autrui et agressé. Le thème du sang et de la renaissance apparaissent également.

Nous terminerons ce parcours des différents univers signifiants convoqués par le conte avec un texte de l'anglais Dante Gabriel Rossetti, poète et peintre préraphaélite. Il s'agit d'un poème intitulé "Jenny ${ }^{18}$ dans lequel un homme médite devant une prostituée endormie répondant à ce même prénom. Dans un passage du poème, celle-ci est 
comparée à un crapaud emprisonné dans une pierre et qui vit à travers les siècles sans avoir jamais vu le soleil se lever. Il est sourd, aveugle et solitaire.

Like a toad within a stone

Seated while Time crumbles on ;

Which sits there since the earth was curs'd

For Man's trangression at the first ;

Which, living through all centuries,

Not once has seen the sun arise ;

Whose life, to its cold circle charmed,

The earth's whole summers have not warmed ;

Which always - whitherso the stone

Be flung - sits there, deaf, blind, alone ;-

Aye, and shall not be driven out

Till that which shuts him round about

Break at the very Master's stroke,

And the dust thereof vanish as smoke,

And the seed of Man vanish as dust :-

Even so within this world is Lust ${ }^{19}$.

Le rapprochement entre la femme et le crapaud éclair d'un nouveau jour un épisode mystérieux du conte de Cortázar. Lorsque Jacobo sort du Zagreb et suit l'autre cliente du restaurant, il la perd brusquement au détour d'un coin de rue de façon inexpliquée et se retrouve seul en compagnie d'un crapaud:

... se preguntó si no sería mejor seguirla de cerca para estar seguro de que no iba a dar la vuelta a la manzana con su errática torpeza de miope ; se apuró a llegar a la esquina y vio la callejuela mal iluminada y vacía. Las dos largas tapias de piedra sólo mostraban un portón a la distancia, donde la turista no había podido llegar ; sólo un sapo exaltado por la lluvia cruzaba a saltos de una acera a otra. (p. 171-172.)

Si l'on tient compte du fait que cette femme est vraisemblablement anglaise - our Jacobo cela ne fait aucun doute - et qu'elle semble s'appeler Jenny,

- Jenny - ijo la mujer-. Es lo único que pudimos saber de ella cuando la conocimos, alcanzó a decir que se llamaba Jenny, a menos que estuviera llamando a otra ... (p. 173).

Il est tentant de considérer qu'elle s'est purement et simplement métamorphosée en crapaud. D'autant plus que, dans le conte, elle est déjà animalisée explicitement à plusieurs reprises : le terme " topo » apparaît quatre fois. Par ailleurs, on sait que c'est un vampire, or, traditionnellement, les vampires ont la capacité de se transformer en animaux variés : chauve-souris, serpents, sangsue, et autres bêtes visqueuses de toutes sortes. Pourquoi pas en crapaud? A la cécité du crapaud du poème correspond la myopie sursignifiée de l'anglaise du conte (" debía ser miope ", " con toda su miopía ", «mirada de gruesos vidrios", «el pobre topo miope »). Elle est, elle aussi, solitaire, marginalisée par sa condition de vampire et condamnée à hanter les nuits allemandes sans jamais voir le jour. La confrontation entre le conte et le poème est donc très éclairante.

«Reunión con un círculo rojo » est un excellent exemple de multiplication des espaces signifiants : espace diégétique et espaces convoqués - pictural ou littéraire-convergent pour élaborer le sens du texte. Chacun apporte sa contribution à la construction d'un espace fantastique qui n'est peut être pas, cependant, bien loin des réalités latinoaméricaines. Ce recours à des univers signifiants extra-textuels permet de concilier la brièveté, la densité d'une part et l'ouverture interprétative d'autre part. Les contraintes du genre sont donc parfaitement respectées. 


\section{NOTES}

1. Alguien que anda por ahí, Madrid : Alfaguara, 1993. Traduction française : Façons de perdre, Paris : Gallimard, 1978.

2. J. Cortázar, «Reunión con un círculo rojo ", Alguien que anda por ahí, Madrid: Alfaguara, 1993, p. 164. Toutes les citations du conte seront suivies dorénavant de l'indication de leur page dans cette édition. C'est nous qui soulignons.

3. G. Bachelard, La poétique de l'espace, Paris : PUF, 1994, p. 131.

4. Ernesto González Bermejo, Conversaciones con Cortázar, Barcelona : EdHASA, 1978, p. 144-145.

5. «lo que llamo intensidad en un cuento consiste en la eliminación de todas las ideas o situaciones intermedias, de todos los rellenos o fases de transición que la novela permite e incluso exige ». Autrement dit, l'intensité s'obtient «mediante la eliminación de todo lo que no converja esencialmente al drama ", J. Cortázar, "Algunos aspectos del cuento", Casa de las Américas, Julio de 1970, p. 183.

6. J. Poulet, «L'espace, élément structurant du fantastique dans Alguien que anda por ahí, Cahiers du G.R.I.A.S., Poétique du déplacement, n4, 1996, p. 190.

7. Ernesto González Bermejo, Op. cit., p. 145.

8. Solferino : de color morado rojizo (DRAE).

9. G. Genette, Palimpsestes, Paris : Seuil, 1982,p.7.

10. Première édition de Alguien que anda por ahí, Madrid : Alfaguara, 1977.

11. Ernesto González Bermejo, op. cit., p. 144.

12. Malva E. Filer, «Reunión con un círculo rojo »: el relato fantástico y la pintura de Jacobo Borges ", América, 17, 1997, p. 87-94.

13. D. Ashton, Jacobo Borges, Caracas : Ediciones Gráficas Armitano, 1983, p. 106.

14. Malava E. Filer, op. cit., p. 92.

15. J. Cortázar, 62 Modelo para armar, Buenos Aires : Editorial Sudamericana, 1968, p. 24.

16. Ibid., p. 17.

17. « ... era también pensar en Frau Marta, en un grito, porque las criaditas de la condesa debían gritar en los sótanos de la Blutgasse, y a la condesa tenía que gustarle que gritaran, si no hubiesen gritado a la sangre le habría faltado ese perfume de heliotropo y marisma ", Ibid., p. 14.

18. Dante Gabriel Rossetti, «Jenny ", Poems \& translations, Oxford: OSA, 1913 (1ère édition), p. 62-73.

19. Ibid.,p. 70, v. 282-297.

\section{AUTEUR}

\section{MARIE-PIERRE LAVAUD}

Université Stendhal-Grenoble III 